\title{
African Ancestry and Its Correlation to Type 2 Diabetes in African Americans: A Genetic Admixture Analysis in Three U.S. Population Cohorts
}

\author{
Ching-Yu Cheng ${ }^{1,4,5,6}$, David Reich ${ }^{7,10}$, Christopher A. Haiman ${ }^{9}$, Arti Tandon ${ }^{7,10}$, Nick Patterson ${ }^{10}$, Selvin \\ Elizabeth $^{1,2}$, Ermeg L. Akylbekova ${ }^{11}$, Frederick L. Brancati ${ }^{1,2,3}$, Josef Coresh ${ }^{1,2}$, Eric Boerwinkle ${ }^{12}$, David \\ Altshuler $^{8,10,13}$, Herman A. Taylor ${ }^{14}$, Brian E. Henderson ${ }^{9}$, James G. Wilson ${ }^{15}$, W. H. Linda Kao ${ }^{1,3 *}$ \\ 1 Department of Epidemiology, Johns Hopkins University, Baltimore, Maryland, United States of America, 2 Welch Center for Prevention, Epidemiology and Clinical \\ Research, Johns Hopkins University, Baltimore, Maryland, United States of America, 3 Department of Medicine, Johns Hopkins University, Baltimore, Maryland, United \\ States of America, 4 Saw Swee Hock School of Public Health, and Department of Ophthalmology, Yong Loo Lin School of Medicine, National University of Singapore, \\ Singapore, Singapore, 5 Duke-NUS Graduate Medical School, Singapore, Singapore, $\mathbf{6}$ Singapore Eye Research Institute, Singapore, Singapore, 7 Department of Genetics, \\ Harvard Medical School, Boston, Massachusetts, United States of America, 8 Department of Medicine, Harvard Medical School, Boston, Massachusetts, United States of \\ America, 9 Department of Preventive Medicine, Keck School of Medicine, University of Southern California, Los Angeles, California, United States of America, 10 Program \\ in Medical and Population Genetics, Broad Institute of Harvard and M.I.T., Cambridge, Massachusetts, United States of America, 11 Jackson Heart Study Analysis Group, \\ Jackson State University, Jackson, Mississippi, United States of America, 12 Human Genetics Center, University of Texas Health Science Center at Houston, Houston, Texas, \\ United States of America, 13 Center for Human Genetic Research and Diabetes Unit, Department of Medicine, Massachusetts General Hospital, Boston, Massachusetts, \\ United States of America, 14 Jackson State University, Tougaloo College, and the University of Mississippi Medical Center, Jackson, Mississippi, United States of America, \\ 15 Department of Physiology and Biophysics, The University of Mississippi Medical Center, Jackson, Mississippi, United States of America
}

\begin{abstract}
The risk of type 2 diabetes is approximately 2 -fold higher in African Americans than in European Americans even after adjusting for known environmental risk factors, including socioeconomic status (SES), suggesting that genetic factors may explain some of this population difference in disease risk. However, relatively few genetic studies have examined this hypothesis in a large sample of African Americans with and without diabetes. Therefore, we performed an admixture analysis using 2,189 ancestry-informative markers in 7,021 African Americans (2,373 with type 2 diabetes and 4,648 without) from the Atherosclerosis Risk in Communities Study, the Jackson Heart Study, and the Multiethnic Cohort to 1) determine the association of type 2 diabetes and its related quantitative traits with African ancestry controlling for measures of SES and 2) identify genetic loci for type 2 diabetes through a genome-wide admixture mapping scan. The median percentage of African ancestry of diabetic participants was slightly greater than that of non-diabetic participants (study-adjusted difference $=1.6 \%, P<0.001)$. The odds ratio for diabetes comparing participants in the highest vs. lowest tertile of African ancestry was 1.33 (95\% confidence interval 1.13-1.55), after adjustment for age, sex, study, body mass index (BMI), and SES. Admixture scans identified two potential loci for diabetes at 12p13.31 (LOD = 4.0) and 13q14.3 ( $\mathrm{Z}$ score $\left.=4.5, P=6.6 \times 10^{-6}\right)$. In conclusion, genetic ancestry has a significant association with type 2 diabetes above and beyond its association with nongenetic risk factors for type 2 diabetes in African Americans, but no single gene with a major effect is sufficient to explain a large portion of the observed population difference in risk of diabetes. There undoubtedly is a complex interplay among specific genetic loci and non-genetic factors, which may both be associated with overall admixture, leading to the observed ethnic differences in diabetes risk.
\end{abstract}

Citation: Cheng C-Y, Reich D, Haiman CA, Tandon A, Patterson N, et al. (2012) African Ancestry and Its Correlation to Type 2 Diabetes in African Americans: A Genetic Admixture Analysis in Three U.S. Population Cohorts. PLoS ONE 7(3): e32840. doi:10.1371/journal.pone.0032840

Editor: Stephen L. Atkin, Postgraduate Medical Institute \& Hull York Medical School, University of Hull, United Kingdom

Received November 9, 2011; Accepted January 31, 2012; Published March 16, 2012

Copyright: (c) 2012 Cheng et al. This is an open-access article distributed under the terms of the Creative Commons Attribution License, which permits unrestricted use, distribution, and reproduction in any medium, provided the original author and source are credited.

Funding: The ARIC Study was carried out as a collaborative study supported by the National Heart, Lung, and Blood Institute (NHLBI) contracts N01-HC-55015, N01-HC-55016, N01-HC-55018, N01-HC-55019, N01-HC-55020, N01-HC-55021 and N01-HC-55022, with support for this analysis by the National Institute of Diabetes and Digestive and Kidney Diseases R21DK073482 and K01DK067207 (WHLK). Genotyping for the ARIC study was carried out by the Johns Hopkins University under federal contract number (N01-HV-48195) from the NHLBI and by the Center for Inherited Disease Research (CIDR). CIDR is fully funded through a federal contract from the United States National Institutes of Health (NIH) to the Johns Hopkins University, Contract Number N01-HG-65403. Research support for JHS studies was provided by R01-HL-084107 (JGW) from the NHLBI and contracts N01-HC-95170, N01-HC-95171, and N01-HC-95172 from the NHLBI and the National Center on Minority Health and Health Disparities. The MEC Study was supported by the National Cancer Institute grants CA63464 and CA54281. DR is supported by a Burroughs Wellcome Career Development Award in the Biomedical Sciences and DR, NP and AT were supported by NIH grant U01-HG004168. FB was supported by NIH grant P60DK079637. The funding sources played no role in design and conduct of the study; collection, management, analysis, and interpretation of the data; and preparation, review, or approval of the manuscript.

Competing Interests: The authors have declared that no competing interests exist.

*E-mail: wkao@jhsph.edu 


\section{Introduction}

Approximately 13\% of the U.S. adults have type 2 diabetes [1], representing a significant burden on public health in the United States. Type 2 diabetes is approximately twice as prevalent in African Americans as in European Americans. In the Multiethnic Cohort (MEC), this racial/ethnic difference persisted after stratification by body mass index (BMI) [2]. Data from the National Health and Nutrition Examination Survey (NHANES) confirm the substantial racial disparity in diabetes across the U.S. [1,3]. In the Atherosclerosis Risk in Communities (ARIC) Study, African Americans are twice as likely as whites to develop incident type 2 diabetes - a disparity which persists even after extensive adjustment for socioeconomic status (SES) and behavioral risk factors [4]. This persistent disparity suggests that genetic factors may contribute to ethnic differences in susceptibility to type 2 diabetes.

Despite remarkable efforts in the past three years that have led to the discovery of more than 30 susceptibility loci for type 2 diabetes and related quantitative traits [5-18], there has been only one genome-wide association study of type 2 diabetes in African Americans [19]. The disparity in diabetes prevalence between Americans of African and European ancestry makes diabetes an attractive phenotype to study by admixture mapping, a method that systematically scans the genomes of groups of recently admixed individuals (e.g., African Americans) to search for genetic loci where persons with a disease or trait, in aggregate, have more (or less) African ancestry than their genome-wide average. Admixture mapping and subsequent fine-mapping studies have been successful in identifying genetic variants for other complex phenotypes, including prostate cancer [20,21], end stage renal disease [22], white blood cell count [23,24], and circulating levels of interleukin 6 soluble receptor [25].

Given the observed ethnic/racial disparities in diabetes prevalence, we hypothesized that some diabetes susceptibility alleles are present at higher frequency in African Americans than in European Americans, resulting in association between genetic ancestry and diabetes risk that is independent of its association with other non-genetic risk factors for type 2 diabetes. Thus we sought 1) to establish the association of genetic ancestry with diabetes and related quantitative traits in African Americans, after accounting for the non-genetic risk factors, and 2) to identify diabetes susceptibility loci by conducting a genome-wide admixture mapping scan. To maximize power to detect genetic association, we performed a pooled analysis of 7,021 AfricanAmerican participants, including 2,373 diabetic cases, from three U.S. population cohorts, including the ARIC Study, the Jackson Heart Study (JHS), and the MEC study.

\section{Results}

\section{Characteristics of participants}

The characteristics and genetic ancestry of the 7,021 African Americans (including 2,373 with type 2 diabetes) included in the study are shown in Table 1 and Figure S1. The overall median global African ancestry was $83.7 \%$ (interquartile range, $76.2 \%-$ $88.7 \%$ ). African ancestry distributions were different among the three cohorts, with MEC participants having a lower average percentage of African ancestry $(P<0.001)$. Diabetic participants tended to have higher BMI, lower education level and lower family income, compared to non-diabetic participants (Table S1).

\section{Association of ancestry with diabetes}

Pooling the three cohorts together, the median percentage of African ancestry of diabetic participants was $1.6 \%$ greater than that of non-diabetic participants $(P<0.001$, adjusted for study). The odd ratios $(\mathrm{ORs})$ for diabetes were higher with increasing tertiles of African ancestry $(P$ for trend $<0.001$, Model 1 in Table 2$)$ after adjustment for age, sex, and study. With additional adjustment for

Table 1. Genetic African ancestry by participant characteristics and study.

\begin{tabular}{|c|c|c|c|c|c|c|c|c|c|}
\hline \multirow[b]{2}{*}{ Characteristic } & \multicolumn{3}{|l|}{ ARIC } & \multicolumn{3}{|l|}{ JHS } & \multicolumn{3}{|l|}{ MEC } \\
\hline & No. (\%) & $\begin{array}{l}\text { African Ancestry, } \\
\text { Median (IQR), \% }\end{array}$ & $P$ Value $^{\mathrm{a}}$ & No. (\%) & $\begin{array}{l}\text { African Ancestry, } \\
\text { Median (IQR), \% }\end{array}$ & $P$ Value $^{\mathrm{a}}$ & No. (\%) & $\begin{array}{l}\text { African Ancestry, } \\
\text { Median (IQR), \% }\end{array}$ & $P$ Value $^{\mathrm{a}}$ \\
\hline Overall & $2285(100)$ & 84.9 (77.8-89.5) & & $3185(100)$ & 84.0 (77.9-88.7) & & $1551(100)$ & $80.6(69.8-87.4)$ & \\
\hline \multicolumn{10}{|l|}{ Age, y } \\
\hline $21-39$ & $0(0)$ & & & $237(7.4)$ & 84.1 (79.4-87.9) & & $0(0)$ & & \\
\hline $40-59$ & 1741 (76.2) & $85.0(78.2-89.4)$ & 0.872 & 1519 (47.7) & 83.9 (77.9-88.3) & 0.455 & $627(40.4)$ & 82.0 (72.4-87.9) & $<0.001$ \\
\hline$\geq 60$ & $544(23.8)$ & 84.6 (76.4-89.9) & & 1429 (44.9) & $84.2(77.4-89.2)$ & & $924(59.6)$ & 79.3 (67.5-86.9) & \\
\hline \multicolumn{10}{|l|}{ Gender } \\
\hline Men & $918(40.2)$ & 85.1 (78.5-89.6) & 0.187 & $1210(38.0)$ & $83.8(77.6-88.2)$ & 0.100 & 1001 (64.5) & $79.2(67.5-86.7)$ & $<0.001$ \\
\hline Women & 1367 (59.8) & $84.7(77.4-89.3)$ & & 1975 (62.0) & $84.2(78.2-88.8)$ & & $550(35.5)$ & $82.2(73.7-88.3)$ & \\
\hline \multicolumn{10}{|l|}{ BMI, $\mathrm{kg} / \mathrm{m}^{2}$} \\
\hline$<25$ & $530(23.2)$ & $83.9(76.8-89.0)$ & & 403 (12.6) & $84.0(78.2-88.8)$ & & 277 (17.8) & 78.9 (67.9-85.8) & \\
\hline $25-<30$ & 865 (37.9) & 84.1 (76.8-89.2) & $<0.001$ & $1053(33.1)$ & $83.8(77.0-88.5)$ & 0.286 & $682(44.0)$ & $79.2(67.2-87.2)$ & $<0.001$ \\
\hline$\geq 30$ & $890(38.9)$ & 85.9 (79.1-89.9) & & $1729(54.3)$ & $84.3(78.3-88.7)$ & & $592(38.2)$ & $82.1(73.4-88.3)$ & \\
\hline \multicolumn{10}{|l|}{ Diabetes } \\
\hline Yes & $631(27.6)$ & 85.7 (79.3-89.5) & 0.008 & $829(26.0)$ & $85.3(79.5-89.3)$ & $<0.001$ & 913 (58.9) & $81.3(71.8-88.1)$ & $<0.001$ \\
\hline No & $1654(72.4)$ & $84.6(77.0-89.4)$ & & $2356(74.0)$ & $83.6(77.3-88.3)$ & & $638(41.1)$ & $79.4(66.2-86.1)$ & \\
\hline
\end{tabular}

ARIC, the Atherosclerosis Risk in Communities Study; JHS, the Jackson Heart Study; MEC, the Multiethnic Cohort; IQR, interquartile range; BMI, body mass index (calculated as weight in kilograms divided by height in meters squared).

${ }^{\text {a }} P$ value was generated from the Wilcoxon rank-sum test or the Kruskal-Wallis test.

doi:10.1371/journal.pone.0032840.t001 
Table 2. Odds ratio of diabetes by genetic African ancestry.

\begin{tabular}{|c|c|c|c|c|c|c|}
\hline \multirow[b]{2}{*}{ Study and Model } & \multicolumn{3}{|c|}{ African Ancestry } & \multirow[b]{2}{*}{$P$ Value for Trend } & \multicolumn{2}{|c|}{ Excess Odds Explained, \% } \\
\hline & Tertile 1 & Tertile 2 & Tertile 3 & & Tertile 2 & Tertile 3 \\
\hline \multicolumn{7}{|c|}{ ARIC, JHS and MEC combined } \\
\hline African ancestry, \% & $<79.4$ & $79.4-87.2$ & $>87.2$ & & & \\
\hline Diabetes, yes/no, No. & $774 / 1574$ & $763 / 1612$ & $836 / 1462$ & & & \\
\hline Model 1, base ${ }^{b}$ & 1 [Reference] & $1.25(1.10-1.43)^{f}$ & $1.48(1.29-1.69)^{9}$ & $<0.001$ & [Reference] & [Reference] \\
\hline Model 2, BMI ${ }^{\mathrm{C}}$ & 1 [Reference] & $1.21(1.06-1.39)^{f}$ & $1.40(1.22-1.61)^{\mathrm{g}}$ & $<0.001$ & 16.0 & 16.7 \\
\hline \multicolumn{7}{|l|}{ ARIC and JHS combined } \\
\hline African ancestry, \% & $<80.5$ & $80.5-87.5$ & $>87.5$ & & & \\
\hline Diabetes, yes/no, No. & $296 / 1088$ & $255 / 1000$ & $406 / 962$ & & & \\
\hline Model 1, base ${ }^{\mathrm{b}}$ & 1 [Reference] & $1.35(1.16-1.57)^{\mathrm{g}}$ & $1.47(1.26-1.71)^{g}$ & $<0.001$ & [Reference] & [Reference] \\
\hline Model 2, BMI ${ }^{\mathrm{C}}$ & 1 [Reference] & $1.32(1.17-1.70)^{f}$ & $1.40(1.20-1.64)^{\mathrm{g}}$ & $<0.001$ & 8.6 & 14.9 \\
\hline Model 3, SES ${ }^{d}$ & 1 [Reference] & $1.27(1.09-1.49)^{f}$ & $1.37(1.17-1.59)^{\mathrm{g}}$ & $<0.001$ & 22.9 & 21.3 \\
\hline Model 4, BMI+SES e & 1 [Reference] & $1.26(1.07-1.48)^{f}$ & $1.33(1.13-1.55)^{\mathrm{g}}$ & $<0.001$ & 25.7 & 29.8 \\
\hline
\end{tabular}

ARIC, the Atherosclerosis Risk in Communities Study; JHS, the Jackson Heart Study; MEC, the Multiethnic Cohort; BMI, body mass index (calculated as weight in kilograms divided by height in meters squared); SES, socioeconomic status (including education, income and occupation).

a Excess risk explained is defined as $\left(\theta_{1}-\theta_{2}\right) /\left(\theta_{1}-1\right)$ where $\theta_{1}$ is the odds ratio of diabetes due to increase in African ancestry in Model $1 ; \theta_{2}$ is the odds ratio after additional adjustment for covariates in each model; and $\theta_{1}-1$ is the excess odds of diabetes due to increase in African ancestry.

${ }^{\mathrm{b}}$ Model 1: odds ratio (95\% confidence interval) is adjusted for age, sex, and study.

cModel 2: odds ratio (95\% confidence interval) is adjusted for covariates in Model 1 and BMI.

${ }^{\mathrm{d}}$ Model 3: odds ratio (95\% confidence interval) is adjusted for covariates in Model 1 and SES.

${ }^{\mathrm{e}}$ Model 4: odds ratio (95\% confidence interval) is adjusted for covariates in Model 1, BMI, and SES.

${ }^{f} P<0.05$, as compared to the reference tertile.

${ }^{g} P<0.001$, as compared to the reference tertile.

doi:10.1371/journal.pone.0032840.t002

BMI, individuals in the second and third tertiles were still, respectively, 1.21 and 1.4 times more likely to have diabetes than their counterparts in the first tertile (Model 2 in Table 2).

To determine whether the observed excess odds of diabetes with increasing African ancestry might further be explained through the association between genetic ancestry and other non-genetic risk factors, such as measures of SES, we constructed additional models using only ARIC and JHS, where these data were available. Univariately, measures of SES were associated with both genetic ancestry (Table S2) and diabetes (Table S3). Even after adjusting for the three SES indicators, individuals in the second and third tertiles of African ancestry were about 1.27 and 1.37 times more likely to have diabetes than those in the first tertile (Model 3 in Table 2). The three SES indicators together accounted for about $22 \%$ of the excess odds of diabetes with increasing African ancestry. Collectively, BMI and the three SES measures explained about $30 \%$ of the excess odds of diabetes observed in individuals in the third tertile. The associations between increasing African ancestry and greater odds of diabetes were also evident in models based on restricted cubic splines (Figure 1).

\section{Association of ancestry with diabetes-related traits}

In ARIC and JHS only, we assessed the association of African ancestry to hemoglobin $\mathrm{A}_{1 \mathrm{c}}\left(\mathrm{HbA}_{1 \mathrm{c}}\right)$, fasting glucose and insulin level, and insulin resistance, which was estimated by the homeostasis model assessment (HOMA-IR) (Table S4 and Table S5). A total of 4,880 participants had measurements on $\mathrm{HbA}_{1 \mathrm{c}}$, and 5,037 had fasting glucose and insulin level. Greater African ancestry was significantly correlated with higher $\mathrm{HbA}_{1 \mathrm{c}}(P<0.001$, see also Figure S2). However, African ancestry accounts for only $0.7 \%$ of the variance in $\mathrm{HbA}_{1 \mathrm{c}}$ levels after adjustment for age, sex and study, while SES alone accounted for a slightly higher proportion of variance of $1.1 \%$. After excluding individuals who were receiving diabetes treatment (because such treatment directly affects trait levels), the effects of ancestry generally became weaker (Table 3). The other three traits, fasting glucose, insulin and HOMA-IR levels, were also positively associated with African

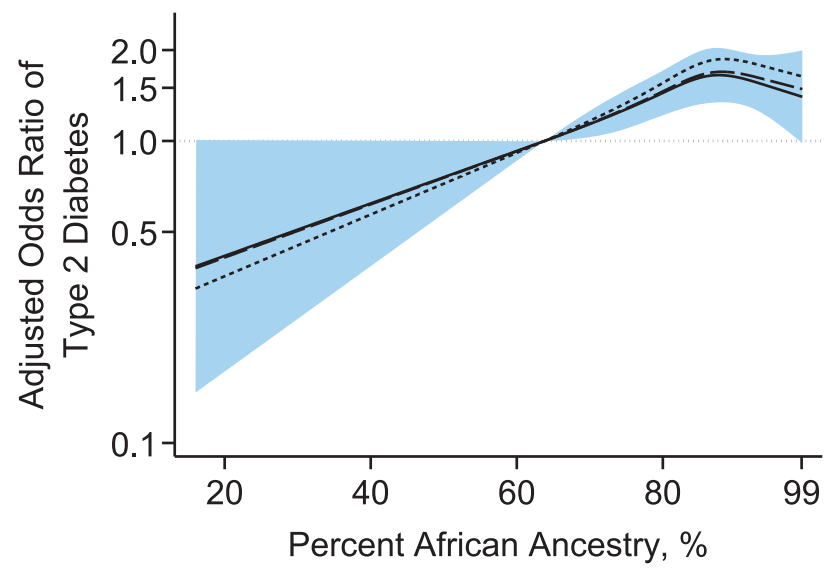

Figure 1. Odds ratio of type 2 diabetes by African ancestry in the ARIC and JHS studies. Odds ratios were based on restricted cubic splines with knots at the 5th, 35th, 65th and 95th percentiles. The reference was set at the 5 th percentile $(63.8 \%)$ of the African ancestry distribution. The odds ratio was adjusted for age, sex and study (shotdashed line), and further adjusted for socioeconomic status, including education level, family income, and occupations (long-dashed line). The solid line indicates the odds ratio adjusted for age, sex, study, socioeconomic status, and $\mathrm{BMI}$; the shaded area represents its $95 \%$ confidence intervals.

doi:10.1371/journal.pone.0032840.g001 
Table 3. Mean difference in the levels of diabetes-related quantitative traits by genetic African ancestry after excluding participants receiving diabetes treatment.

\begin{tabular}{|c|c|c|c|c|c|c|}
\hline \multirow[t]{2}{*}{ Trait and Model } & \multicolumn{3}{|c|}{ African Ancestry ${ }^{a}$} & \multirow[t]{2}{*}{$P$ Value for Trend } & \multicolumn{2}{|c|}{ Effect Explained, $\%^{\mathbf{b}}$} \\
\hline & Tertile 1 & Tertile 2 & Tertile 3 & & Tertile 2 & Tertile 3 \\
\hline \multicolumn{7}{|c|}{ Hemoglobin $A_{1 c} \%(n=4100)$} \\
\hline Model 1 , base ${ }^{c}$ & 0 [Reference] & $0.10(0.02-0.18)^{9}$ & $0.10(0.02-0.18)^{9}$ & 0.011 & [Reference] & [Reference] \\
\hline Model 2, BMI ${ }^{\mathrm{d}}$ & 0 [Reference] & $0.08(0.01-0.16)^{9}$ & $0.09(0.01-0.17)^{9}$ & 0.028 & 20.0 & 10.0 \\
\hline Model 3, SES $\mathrm{e}^{\mathrm{e}}$ & 0 [Reference] & $0.07(-0.01-0.16)$ & $0.07(-0.01-0.16)$ & 0.073 & 30.0 & 30.0 \\
\hline Model 4, BMI+SES ${ }^{f}$ & 0 [Reference] & $0.06(-0.01-0.14)$ & $0.06(-0.02-0.14)$ & 0.122 & 40.0 & 40.0 \\
\hline \multicolumn{7}{|c|}{ Glucose, mg/dL ( $n=4423)$} \\
\hline Model 1, base ${ }^{c}$ & 0 [Reference] & $1.20(-0.89-3.28)$ & $1.57(-0.51-3.65)$ & 0.139 & [Reference] & [Reference] \\
\hline Model 2, BMI ${ }^{\mathrm{d}}$ & 0 [Reference] & $0.93(-1.14-2.99)$ & $1.16(-0.91-3.22)$ & 0.272 & 22.5 & 26.1 \\
\hline Model 3, SES & 0 [Reference] & $0.48(-1.62-2.58)$ & $0.73(-1.38-2.84)$ & 0.501 & 60.0 & 53.5 \\
\hline Model 4, BMI+SES ${ }^{f}$ & 0 [Reference] & $0.30(-1.80-2.38)$ & $0.41(-1.68-2.51)$ & 0.700 & 75.8 & 73.9 \\
\hline \multicolumn{7}{|l|}{ Insulin, mU/L ( $n=4423)$} \\
\hline Model 1, base ${ }^{c}$ & 0 [Reference] & $0.87(0.12-1.62)^{9}$ & $0.68(-0.07-1.43)$ & 0.073 & [Reference] & [Reference] \\
\hline Model 2, BMI ${ }^{\mathrm{d}}$ & 0 [Reference] & $0.58(-0.11-1.27)$ & $0.23(-0.46-0.93)$ & 0.503 & 33.3 & 66.2 \\
\hline Model 3, SES & 0 [Reference] & $0.81(0.06-1.57)^{9}$ & $0.60(-0.16-1.36)$ & 0.125 & 6.9 & 11.8 \\
\hline Model 4, BMI+SES ${ }^{f}$ & 0 [Reference] & $0.60(-0.10-1.29)$ & $0.25(-0.46-0.95)$ & 0.498 & 31.0 & 63.2 \\
\hline \multicolumn{7}{|l|}{ HOMA-IR $(n=4423)$} \\
\hline Model 1, base ${ }^{c}$ & 0 [Reference] & $0.28(0.05-0.52)^{9}$ & $0.23(-0.00-0.47)$ & 0.052 & [Reference] & [Reference] \\
\hline Model 2, BMI ${ }^{\mathrm{d}}$ & 0 [Reference] & $0.20(-0.02-0.42)$ & $0.10(-0.12-0.32)$ & 0.352 & 28.6 & 56.5 \\
\hline Model 3, SES & 0 [Reference] & $0.24(-0.00-0.47)$ & $0.17(-0.07-0.41)$ & 0.159 & 14.3 & 26.1 \\
\hline Model 4, BMI+SES ${ }^{f}$ & 0 [Reference] & $0.17(-0.05-0.40)$ & $0.07(-0.15-0.30)$ & 0.536 & 39.3 & 69.6 \\
\hline
\end{tabular}

BMI, body mass index (calculated as weight in kilograms divided by height in meters squared); SES, socioeconomic status (including education, income and occupation). ${ }^{a}$ Tertiles 1,2 and 3 of African ancestry are $<80.3 \%, 80.3 \%-87.3 \%$ and $>87.3 \%$, respectively, for hemoglobin $\mathrm{A}_{1 \text { c }}$, and $<80.2 \%, 80.2 \%-87.3 \%$ and $>87.3 \%$, respectively, for glucose, insulin, and HOMA-IR.

${ }^{\mathrm{b}}$ Effects explained is defined as $\left(\beta_{1}-\beta_{2}\right) / \beta_{1}$ where $\beta_{1}$ is the regression coefficient of traits in Model $1 ; \beta_{2}$ is the regression coefficient after adjustment for covariates in each model.

${ }^{c}$ Model 1: Mean difference (95\% confidence interval) is adjusted for age, sex, and study.

${ }^{\mathrm{d}}$ Model 2: Mean difference (95\% confidence interval) is adjusted for covariates in Model 1 and BMI.

Model 3: Mean difference (95\% confidence interval) is adjusted for covariates in Model 1 and SES.

f Model 4: Mean difference (95\% confidence interval) is adjusted for covariates in Model 1, BMI, and SES.

${ }^{9} P<0.05$, as compared to the reference tertile.

doi:10.1371/journal.pone.0032840.t003

ancestry before and after adjustment, but the associations were not statistically significant (Table 3 and Table S5).

\section{Admixture scans}

We conducted genome-wide admixture scans on type 2 diabetes in the 7,021 African Americans (Figure 2 and Table S6). In the diabetic cases, we detected an admixture association in diabetic cases at 12p13.31 with a locus-specific LOD of 4.0, just reaching the threshold for suggestiveness. The $12 \mathrm{p} 13.31$ peak was also supported by a case-control $\mathrm{Z}$ score of -4.2 (nominal $P=$ $3.3 \times 10^{-5}$ ), which was marginally genome-wide significant. At this locus, diabetic cases had lower European ancestry (i.e., higher African ancestry) than non-diabetic controls. The second strongest admixture signal was observed nearby at 12q13.13 (locus-specific $\mathrm{LOD}=3.8$ ), and the third was at 1 p33 (locus-specific LOD = 3.5). There were no other loci with LOD scores $>2.5$. Averaging the LOD scores across all loci in the genome, we obtained a genomewide score of 1.5, again reaching the threshold of 1 for suggestiveness. Interestingly, at 13q14.3 the LOD scores was far from significant (locus-specific LOD = 1.1), but this locus had both the largest magnitude (either positive or negative) case-control statistic anywhere in the genome $(\mathrm{Z}$ score $=4.5$, nominal
$P=6.6 \times 10^{-6}$ ), exceeding the level of nominal genome-wide significance. At the 13q14.3 locus, the diabetic cases had higher European ancestry than the non-diabetic controls.

\section{Discussion}

We have conducted a large-scale admixture genetic analysis in more than 7,000 African Americans to determine the association of African ancestry with type 2 diabetes and to map susceptibility loci for type 2 diabetes. With 2,373 cases with type 2 diabetes and 4,648 controls, we found that greater African ancestry was significantly associated with type 2 diabetes and $\mathrm{HbA}_{1 \mathrm{c}}$ values even after adjustment for BMI and markers of SES, including education, income, and occupation. Despite the significant association between greater African ancestry and type 2 diabetes, no major locus for diabetes could be detected by our admixture scans, using the more powerful locus-genome statistic.

Our results show that there is $30 \%$ to $40 \%$ increase in odds of type 2 diabetes among participants in the highest $(>87.5 \%)$ vs. the lowest $(<80.5 \%)$ tertile of African ancestry, even after adjustment for measures of SES and/or BMI. Our restricted cubic spline models also support this extrapolation, implying that genetic ancestry is a major independent determinant of the observed 


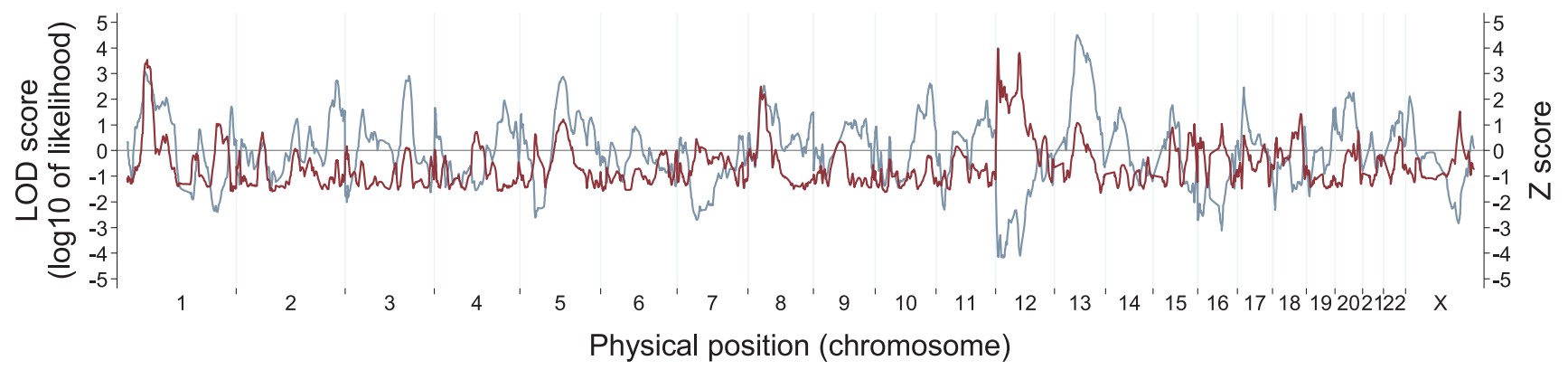

Figure 2. Admixture scans for genetic loci of type 2 diabetes in African Americans. Locus-genome statistic (LOD score, red line) and casecontrol statistic (Z score, blue gray line) are shown. A signal was detected at 12p13.31 with a locus-specific LOD score of 4.0, just reaching the threshold of 4 for suggestiveness. The 12p13.31 peak was also supported by the case-control statistic $\left(Z\right.$ score $=-4.2$, nominal $P=3.3 \times 10^{-5}$ ). The second strongest admixture signal was observed on the same chromosome at 12q13.13 (locus-specific LOD =3.8). There was also an admixture peak at 13q14.3 that did not reach genome-wide significance by the locus-genome statistic (locus-specific LOD =1.1), but that had the largest magnitude case-control $Z$ score anywhere in the genome $\left(Z\right.$ score $=4.5$, nominal $\left.P=6.6 \times 10^{-6}\right)$.

doi:10.1371/journal.pone.0032840.g002

disparity in diabetes risk between the two ethnic groups. We note that in our study, markers of SES (education, income, and occupation) account for only a modest proportion $(\sim 22 \%)$ of the excess odds of type 2 diabetes due to ancestry. The results contrast to previous findings in Hispanic Americans [26], where the association between their non-European ancestry and type 2 diabetes is also significant, but where SES appears to be a much greater confounder, as adjustment for it significantly attenuated the association signal. It is also worth noting that the previous study had less power than our study as it used fewer individuals and fewer ancestry informative markers [26].

Insulin resistance and $\beta$-cell dysfunction are known to be major factors in the pathogenesis of type 2 diabetes. Evidence from epidemiological studies indicates that African Americans tend to be more insulin resistant and have greater insulin responses to glucose than European Americans [27-33]. Our results showed a positive (but statistically non-significant) correlation between African ancestry and HOMA-IR, in line with one earlier study that demonstrated that children with greater African ancestry had lower insulin sensitivity and a higher acute insulin response [34]. In genome-wide scans to date, the majority of the genetic variants for type 2 diabetes identified in European-derived populations appeared to be related to impaired insulin secretion $[6,17,35]$, while only IRS1 has been unequivocally associated with insulin resistance [18]. However, a limitation is that these studies have been carried out largely in Europeans. It will be interesting to explore whether the loci associated to type 2 diabetes in African Americans are also associated with impaired insulin secretion, once genome-wide association studies of sufficient power are carried out.

Previous genome-wide scans for type 2 diabetes in Africandescent populations have been extremely limited, and there has only been one study using admixture-based approaches. In the GENNID (Genetic of NIDDM) Study, using markers from a linkage panel in 1,450 African Americans, the strongest admixture association was found on chromosome $12(90 \mathrm{cM})$, but no loci achieved genome-wide significance [36]. In our large population with a high number of ancestry informative markers, the two most interestingly loci using were at 12 p13.31 and 13q14.3. While neither of these loci was significant by our locus-genome statistic which has the most statistical power, the case-control $\mathrm{Z}$ score at both loci exceeded the threshold for genome-wide significance, which makes these loci of interest for further study. An attractive candidate gene at the $12 \mathrm{p} 13.31$ locus is glyceraldehyde-3- phosphate dehydrogenase $(G A P D H)$, which is a key enzyme in the glycolytic pathway and is known to affect insulin receptor signaling [37]. The 12p13.31 locus has been found to be associated with type 1 diabetes in previous genome-wide association studies in European-derived populations [15], but neither of the two loci has been associated with type 2 diabetes in either African Americans or Europeans.

An interesting feature of our admixture scanning results is that diabetes risk at $12 \mathrm{p} 13.31$ and $13 \mathrm{q} 14.3$ were associated with ancestry in opposite directions. At 13q14.3, greater European ancestry is associated with a higher risk of diabetes, opposite to the direction of the overall epidemiological association, a phenomenon that we documented for the first time in a study of obesity loci [38]. These two loci, together with the other modest admixture signals on chromosome 12 and 1 , and the absence of significant signals in locus-genome statistic elsewhere in the genome, suggest no evidence for a large genetic effect for type 2 diabetes that is racially/ethnically differentiated, such as that at the MYH9/ApoL1 locus for non-diabetic end stage renal disease [22,39]. Thus multiple loci modest effects may, in aggregate, explain the apparent difference in genetic risk for type 2 diabetes between African Americans and European Americans.

Our study has important limitations. Despite the fact that our study size far exceeds previous genome scans for type 2 diabetes risk loci in African Americans, statistical power remains an important concern. We carried out simulation studies to examine the power of our study to detect a genomic locus of elevated African ancestry [40]. With a total of 2,373 diabetic cases, we expect to have $80 \%$ power to detect a 1.8 -fold increased risk of type 2 diabetes per allele for alleles that are ancestry informative between Europeans and West Africans but less power for weaker ORs. A second limitation is that we used BMI as the only measure of adiposity. Including some other measure, such as waist circumference, might further attenuate the diabetes-ancestry association. In our previous analysis, however, we found that BMI, but not waist circumference, was significantly correlated with genetic ancestry after adjustment for SES [41], suggesting that confounding by waist circumference would have a minimal effect on results. Third, as in many studies involving SES, we were not able to fully assess SES and made inferences about SES based on education, income, and occupation, which, although are strong markers for SES, are still imperfect [42]. For example, SES may also be correlated with other diabetes risk factors, such as diet and life-style, and historical socioeconomic factors could in theory 
interact in complex ways with African ancestry to influence diabetes risk, making the associations among them even more complicated.

In summary, in community-based populations with more than 7,000 African Americans, we found that genetic ancestry is significant associated with type 2 diabetes above and beyond the effects of markers of SES, and we detected several suggestive loci that may harbor genetic variants modulating diabetes risk. These results suggest that in African Americans, genetic ancestry has a significant effect on the risk of type 2 diabetes that are independent of the contribution of SES, but that no single locus with a major effect explains a large portion of the observed disparity in diabetes risk between African Americans and European Americans. In addition, they suggest that genetic measured African ancestry contributes to the risk of type 2 diabetes via both genetic and nongenetic pathways. The effect of ancestry on any individual locus in the genome is likely to be modest, but in aggregate, differences in ancestry may contribute substantially to the observed ethnic disparity in risk of type 2 diabetes.

\section{Materials and Methods}

\section{Ethics statement}

This study was conducted according to the principles expressed in the Declaration of Helsinki. All data collections were carried out according to protocols approved by Johns Hopkins Bloomberg School of Public Health Institutional Review Board for the study of human subjects. Written informed consent was obtained from all participants.

\section{Study populations}

The individuals enrolled in the present study came from three studies: the ARIC, JHS and MEC studies (Table 1). A detailed description of the three studies as well as the numbers of participants that were analyzed after applying various data quality filters are presented in Text S1. A brief description of each study is provided here.

The ARIC study is a prospective epidemiologic study that examines clinical and subclinical atherosclerotic disease in a cohort of 15,792 persons, including 4,266 self-reported African Americans, aged 45 to 64 years at their baseline examination from 1987 to 1989. The sampling procedure and methods used in ARIC have been described in detail elsewhere [43]. A total of 2,285 African-American participants from the ARIC study were included in the current analysis.

The JHS is a long-term, community-based observational study of cardiovascular disease and its risk factors in 5,301 self-identified African Americans recruited between 2000 and 2004 from three counties surrounding Jackson, Mississippi [44,45]. Unrelated persons aged $35-84$ were enrolled, and the remaining participants, at least 21 years old, were members of the nested JHS Family Study [46]. A total of 3,185 participants from the JHS were included in the current study.

The MEC study is a prospective cohort of 215,251 individuals recruited between 1993 and 1996 in Hawaii and Los Angeles, California, of whom 16.3\% were African Americans [47]. Potential cohort members were identified primarily through Department of Motor Vehicles drivers' license files and, additionally for African Americans, Health Care Financing Administration data files. Participants were between the ages of 45 and 75 years at the time of recruitment. A total of 1,551 African-American participants from the MEC study, selected for a diabetes case-control study, were included in this analysis.

\section{Diabetes and related traits}

Information on body weight and height was collected in all three studies. In ARIC and JHS, anthropometry was performed during the clinical visit in the fasting state with an empty bladder by certified technicians. Body mass index was calculated as weight (in $\mathrm{kg}$ )/height (in meters) squared. In MEC, BMI was calculated using self-reported weight and height. The ARIC Study and JHS also have measurements of other diabetes-related quantitative traits, including fasting serum glucose and insulin concentrations, and $\mathrm{HbA}_{1 \mathrm{c}}$. Participants were asked to fast for at least 12 hours before morning blood collection. Blood samples were collected into vacuum tubes containing serum-separator gel (glucose, insulin) or EDTA $\left(\mathrm{HbA}_{1 \mathrm{c}}\right)$. Specimens were then processed and analyzed in the ARIC and JHS Central Laboratories at University of Minnesota [48,49]. Serum glucose and insulin were measured by the hexokinase method [49,50] and by radioimmunoassay [49], respectively. $\mathrm{HbA}_{1 \mathrm{c}}$ was assayed with Tosoh HPLC instruments $[48,49]$. The present analysis used data from the baseline examination in all three cohorts, except that in ARIC $\mathrm{HbA}_{1 \mathrm{c}}$ was measured in stored whole blood samples from the second clinical visit [48]. Insulin resistance was estimated by the homeostasis model assessment (HOMA-IR) as fasting plasma glucose $[\mathrm{mmol} / \mathrm{l}]$ times fasting serum insulin $[\mathrm{mU} / \mathrm{L}]$ divided by 22.5.

Type 2 diabetes was defined as the presence of any one of the following at the baseline examination in the ARIC and JHS studies: 1) fasting glucose $\geq 7.0 \mathrm{mmol} / \mathrm{l}(126 \mathrm{mg} / \mathrm{dl})$; 2) nonfasting glucose $\geq 11.1 \mathrm{mmol} / \mathrm{l}(200 \mathrm{mg} / \mathrm{dl})$; 3) hemoglobin $\mathrm{A}_{\mathrm{lc}}$ $\geq 6.5 \%$ [51]; 4) current use of diabetic medication; or 5) a positive response to the question "Has a doctor ever told you that you had diabetes (sugar in the blood)?" In addition, diabetic individuals in ARIC or JHS who reported age of diagnosis younger than 30 years were excluded. In the MEC, diabetic individuals were defined as those who indicated on the baseline or follow-up questionnaires that they had a history of diabetes, and were taking medication for diabetes at the time of blood draw. The question did not differentiate between type 1 diabetes and type 2 diabetes, and thus we expect a small fraction $(<10 \%)$ of the respondents to have type 1 diabetes [52]. In the ARIC and JHS study, nondiabetic controls were defined as African-American participants who did not have diabetes and were derived from the same populations as the diabetic cases. In the MEC, non-diabetic controls were from a group of samples who neither had a history of diabetes nor were taking medication for diabetes and had been specifically genotyped as part of previous admixture scans for prostate cancer [20] and hypertension [53].

\section{Socioeconomic status}

Information on three SES indicators, including personal education level, occupation, and family income, was collected during the baseline interview in the ARIC and JHS study. For the purpose of this analysis, education level was categorized into four groups: 1) less than high school; 2) high school graduate or high school-level General Educational Development credential; 3) some college; or 4) college completed, or some graduate or professional school. Income level was categorized as affluent, upper-middle, lower-middle, or poor based on total combined family income, family size, and poverty levels in each year when the interview was conducted. Some participants $(9.5 \%$ and $13.8 \%$ in ARIC and JHS, respectively) did not provide their income information and were coded as a separate category (missing). A more detailed description of the assessment of income is presented in Text S1. Occupations were categorized according to the criteria of the 1980 U.S. census into six groups: managerial and professional specialty; 
sales, technical, and administrative support; service; farming, forestry and fishing or precision production; operators, fabricators, laborers or construction; and homemakers [54]. Because cohort controls had not been genotyped in the MEC (as we had oversampled particular phenotypes for genetic studies), the analyses of SES was limited to ARIC and JHS only.

\section{Genotyping}

Participants were genotyped with at least one of three iteratively improved and partially overlapping panels of ancestry-informative SNP markers $[23,25,41,55,56]$. The ARIC study used the Phase 3 panel, the JHS study used Phase 2 and 3, and the MEC study used all three panels. Altogether 2,189 markers were genotyped in the present study, with a median of 1,243 markers per individual. We used previously published genotyping data to estimate the frequency of each SNP in West Africans and European Americans, the two parental populations of African Americans $[20,53,55]$. A series of filters, as described previously [25,55-57], were applied to detect and remove SNPs with problematic genotyping. Genotyping details, estimates of allele frequencies, and SNP quality control checks are presented in Text S1.

\section{Estimating genetic ancestry}

We estimated each subject's global percentage of African ancestry using the ANCESTRYMAP software [40]. ANCESTRYMAP uses a Hidden Markov Model (HMM) to combine the weak information about local ancestry that is provided by each SNP into a more confident estimate that incorporates information from many neighboring markers. Use of the HMM to estimate genetic ancestry is described in more detail in Text $\mathrm{S} 1$.

\section{Statistical analysis}

Statistical analyses were performed using Stata 10.1 (Stata Corporation, College Station, Texas, US) and ANCESTRYMAP. Initial analyses of the correlation between African ancestry and either SES or the diabetes-related quantitative traits were performed using Spearman's correlation coefficient $(\rho)$. Analyses of ancestry associations were conducted using pooled data from all three cohorts. Quantitative traits were available for analysis in ARIC and JHS only.

We used logistic regression models to estimate the OR for diabetes, comparing tertiles 2 and 3 to the lowest tertile of the distribution of African ancestry. To explore further potential nonlinear ancestry-diabetes associations, we used restricted cubic splines with equally-spaced knots at the 5th, 35th, 65th, and 95th percentiles of the ancestry distribution. For quantitative traits, we used linear regression models to determine the proportion of variation in trait levels explained by each variable (i.e., the coefficient of determination) and to assess the change in trait levels with ancestry tertiles. We constructed a series of multivariate models for our regression analyses. The base models were adjusted for age, sex and study only. Subsequent models were created by introducing BMI and SES as covariates, separately and collectively in sequence, because both SES and BMI correlate with ancestry $[38,41]$ and thus may potentially confound ancestry-diabetes association.

To quantify the extent to which groups of covariates appeared to explain the excess odds of diabetes with increasing African ancestry, we calculated the percentage reduction in the OR associated with adjustment (see Text S1 for more details). Similar calculation was performed to determine the relative contribution of covariates to the observed association between ancestry and quantitative traits.
We used ANCESTRYMAP [40] to search for genomic regions associated with an increased percentage of either European or African ancestry. The ANCESTRYMAP software provided two statistics: a locus-genome statistic and a case-control statistic. A locusgenome statistic was obtained in cases by calculating the likelihood of the genotyping data at the SNPs at the locus under a risk model and comparing it to the likelihood of the genotyping data at the SNPs at the locus assuming that the locus is unassociated with the phenotype [40]. We tested 6 pre-specified European ancestry risk models ranging from 0.7 to 1.3 . To accumulate evidence of association in these models, we averaged the ratio of these two likelihoods emerging from each model at each point in the genome, taking the $\log _{10}$ of this likelihood ratio to produce a locusspecific LOD score. We considered a locus-specific LOD score for association at a particular locus of $>5$ as genome-wide significant and $>4$ as suggestive [58]. To obtain an assessment of the evidence for a risk locus anywhere in the genome, we averaged the likelihood ratio for association across all loci in the genome, and took the $\log 10$ to obtain a "genome-wide score" [58]. We interpreted a genome-wide score $>2$ as significant, and $>1$ as suggestive.

A case-control statistic was calculated by comparing locus-specific deviations in European ancestry in cases versus controls at each locus across the genome [40]. For loci identified by the casecontrol statistic, the level of genome-wide significance was defined as a $\mathrm{Z}$ score $>4.06$ or $<-4.06$, corresponding to an uncorrected nominal $P<5 \times 10^{-5}$, or a corrected nominal $P<0.05$ after conservatively correcting for 1,000 hypotheses tested (approximately equals the number of independent chromosomal segments assigned to either African or European ancestry).

\section{Supporting Information}

Figure S1 Histograms of African ancestry in the African American participants by study and diabetes status. Percentages of African ancestry were estimated using subsets of 2,189 ancestry-informative SNPs. The grey bars represent the diabetic participants; the blue bars represent the non-diabetic participants.

(DOC)

Figure S2 Scatterplot of hemoglobin $A_{1 c}$ and percentage of African ancestry in the ARIC and JHS studies. The solid line in the figure is a lowess smoother.

(DOC)

Table S1 Characteristics of ARIC and JHS study participants by diabetes status.

(DOG)

Table S2 Genetic African ancestry by socioeconomic status in African Americans in the ARIG and JHS studies $(\mathbf{n}=\mathbf{5 4 7 0})$.

(DOC)

Table S3 Association of diabetes and socioeconomic status in African Americans in the ARIG and JHS studies.

(DOC)

Table S4 Genetic African ancestry by diabetes-related quantitative traits in African Americans in the ARIG and JHS studies.

(DOG)

Table S5 Mean difference in the levels of diabetesrelated quantitative traits by genetic African Ancestry. (DOG) 
Table S6 Summary of the Admixture scans on type 2 diabetes results by chromosome.

(DOC)

Text S1 Supplementary Methods: study populations, assessment of income, genotyping and quality filters, estimating genetic ancestry with Hidden Markov Models, and calculating contribution of covariates to the observed association.

(DOC)

\section{References}

1. Cowie CG, Rust KF, Ford ES, Eberhardt MS, Byrd-Holt DD, et al. (2009) Full accounting of diabetes and pre-diabetes in the U.S. population in 1988-1994 and 2005-2006. Diabetes Care 32: 287-294.

2. Maskarinec G, Grandinetti A, Matsuura G, Sharma S, Mau M, et al. (2009) Diabetes prevalence and body mass index differ by ethnicity: the Multiethnic Cohort. Ethn Dis 19: 49-55.

3. Cowie CC, Rust KF, Byrd-Holt DD, Eberhardt MS, Flegal KM, et al. (2006) Prevalence of diabetes and impaired fasting glucose in adults in the U.S. population: National Health And Nutrition Examination Survey 1999-2002. Diabetes Care 29: 1263-1268.

4. Brancati FL, Kao WH, Folsom AR, Watson RL, Szklo M (2000) Incident type 2 diabetes mellitus in African American and white adults: the Atherosclerosis Risk in Communities Study. JAMA 283: 2253-2259.

5. Voight BF, Scott LJ, Steinthorsdottir V, Morris AP, Dina C, et al. (2010) Twelve type 2 diabetes susceptibility loci identified through large-scale association analysis. Nat Genet 42: 579-589.

6. Frayling TM (2007) Genome-wide association studies provide new insights into type 2 diabetes aetiology. Nat Rev Genet 8: 657-662.

7. Grant SF, Thorleifsson G, Reynisdottir I, Benediktsson R, Manolescu A, et al. (2006) Variant of transcription factor 7-like 2 (TCF7L2) gene confers risk of type 2 diabetes. Nat Genet 38: 320-323.

8. Zeggini E, Scott LJ, Saxena R, Voight BF, Marchini JL, et al. (2008) Metaanalysis of genome-wide association data and large-scale replication identifies additional susceptibility loci for type 2 diabetes. Nat Genet 40: 638-645.

9. Yasuda K, Miyake K, Horikawa Y, Hara K, Osawa H, et al. (2008) Variants in KCNQ1 are associated with susceptibility to type 2 diabetes mellitus. Nat Genet 40: 1092-1097.

10. Saxena R, Voight BF, Lyssenko V, Burtt NP, de Bakker PI, et al. (2007) Genome-wide association analysis identifies loci for type 2 diabetes and triglyceride levels. Science 316: 1331-1336.

11. Scott LJ, Mohlke KL, Bonnycastle LL, Willer CJ, Li Y, et al. (2007) A genomewide association study of type 2 diabetes in Finns detects multiple susceptibility variants. Science 316: 1341-1345.

12. Sladek R, Rocheleau G, Rung J, Dina C, Shen L, et al. (2007) A genome-wide association study identifies novel risk loci for type 2 diabetes. Nature 445 : 881-885.

13. Steinthorsdottir V, Thorleifsson G, Reynisdottir I, Benediktsson R, Jonsdottir T, et al. (2007) A variant in CDKAL1 influences insulin response and risk of type 2 diabetes. Nat Genet 39: 770-775.

14. Zeggini E, Weedon MN, Lindgren CM, Frayling TM, Elliott KS, et al. (2007) Replication of genome-wide association signals in UK samples reveals risk loci for type 2 diabetes. Science 316: 1336-1341.

15. Wellcome Trust Case Control Consortium (2007) Genome-wide association study of 14,000 cases of seven common diseases and 3,000 shared controls. Nature 447: 661-678.

16. Bouatia-Naji N, Bonnefond A, Cavalcanti-Proenca C, Sparso T, Holmkvist J, et al. (2009) A variant near MTNR1B is associated with increased fasting plasma glucose levels and type 2 diabetes risk. Nat Genet 41: 89-94.

17. Stolerman ES, Florez JC (2009) Genomics of type 2 diabetes mellitus: implications for the clinician. Nat Rev Endocrinol 5: 429-436.

18. Rung J, Cauchi S, Albrechtsen A, Shen L, Rocheleau G, et al. (2009) Genetic variant near IRS1 is associated with type 2 diabetes, insulin resistance and hyperinsulinemia. Nat Genet 41: 1110-1115.

19. Lettre G, Palmer CD, Young T, Ejebe KG, Allayee H, et al. (2011) Genomewide association study of coronary heart disease and its risk factors in 8,090 African Americans: the NHLBI CARe Project. PLoS Genet 7: e1001300.

20. Freedman ML, Haiman CA, Patterson N, McDonald GJ, Tandon A, et al. (2006) Admixture mapping identifies 8q24 as a prostate cancer risk locus in African-American men. Proc Natl Acad Sci U S A 103: 14068-14073.

21. Haiman CA, Patterson N, Freedman ML, Myers SR, Pike MC, et al. (2007) Multiple regions within 8q24 independently affect risk for prostate cancer. Nat Genet 39: 638-644.

22. Kao WH, Klag MJ, Meoni LA, Reich D, Berthier-Schaad Y, et al. (2008) MYH9 is associated with nondiabetic end-stage renal disease in African Americans. Nat Genet 40: 1185-1192.

23. Nalls MA, Wilson JG, Patterson NJ, Tandon A, Zmuda JM, et al. (2008) Admixture mapping of white cell count: genetic locus responsible for lower white

\section{Acknowledgments}

The authors thank the staff and participants of the ARIC, JHS, and MEC studies.

\section{Author Contributions}

Conceived and designed the experiments: CYC DR CAH JW WHK. Performed the experiments: DR CAH EB JW. Analyzed the data: CYG AT NP. Contributed reagents/materials/analysis tools: DR CAH SE ELA FLB JC EB DA HAT BEH JW WHK. Wrote the paper: CYC DR WHK.

blood cell count in the Health ABC and Jackson Heart studies. Am J Hum Genet 82: 81-87.

24. Reich D, Nalls MA, Kao WH, Akylbekova EL, Tandon A, et al. (2009) Reduced neutrophil count in people of African descent is due to a regulatory variant in the Duffy antigen receptor for chemokines gene. PLoS Genet 5: e1000360.

25. Reich D, Patterson N, Ramesh V, De Jager PL, McDonald GJ, et al. (2007) Admixture mapping of an allele affecting interleukin 6 soluble receptor and interleukin 6 levels. Am J Hum Genet 80: 716-726.

26. Florez JC, Price AL, Campbell D, Riba L, Parra MV, et al. (2009) Strong association of socioeconomic status with genetic ancestry in Latinos: implications for admixture studies of type 2 diabetes. Diabetologia 52: 1528-1536.

27. Karter AJ, Mayer-Davis EJ, Selby JV, D'Agostino RB, Jr., Haffner SM, et al. (1996) Insulin sensitivity and abdominal obesity in African-American, Hispanic, and non-Hispanic white men and women. The Insulin Resistance and Atherosclerosis Study. Diabetes 45: 1547-1555.

28. Osei K, Schuster DP (1996) Effects of race and ethnicity on insulin sensitivity, blood pressure, and heart rate in three ethnic populations: comparative studies in African-Americans, African immigrants (Ghanaians), and white Americans using ambulatory blood pressure monitoring. Am J Hypertens 9: 1157-1164.

29. Arslanian S, Suprasongsin C (1996) Differences in the in vivo insulin secretion and sensitivity of healthy black versus white adolescents. J Pediatr 129: 440-443.

30. Gower BA, Nagy TR, Trowbridge CA, Dezenberg C, Goran MI (1998) Fat distribution and insulin response in prepubertal African American and white children. Am J Clin Nutr 67: 821-827.

31. Ku CY, Gower BA, Hunter GR, Goran MI (2000) Racial differences in insulin secretion and sensitivity in prepubertal children: role of physical fitness and physical activity. Obes Res 8: 506-515.

32. Haffner SM, D'Agostino R, Saad MF, Rewers M, Mykkanen L, et al. (1996) Increased insulin resistance and insulin secretion in nondiabetic AfricanAmericans and Hispanics compared with non-Hispanic whites. The Insulin Resistance Atherosclerosis Study. Diabetes 45: 742-748.

33. Goedecke JH, Dave JA, Faulenbach MV, Utzschneider KM, Lambert EV, et al. (2009) Insulin response in relation to insulin sensitivity: an appropriate beta-cell response in black South African women. Diabetes Care 32: 860-865.

34. Gower BA, Granger WM, Franklin F, Shewchuk RM, Goran MI (2002) Contribution of insulin secretion and clearance to glucose-induced insulin concentration in african-american and caucasian children. J Clin Endocrinol Metab 87: 2218-2224.

35. Florez JC (2008) Newly identified loci highlight beta cell dysfunction as a key cause of type 2 diabetes: where are the insulin resistance genes? Diabetologia 51: $1100-1110$.

36. Elbein SC, Das SK, Hallman DM, Hanis CL, Hasstedt SJ (2009) Genome-wide linkage and admixture mapping of type 2 diabetes in African American families from the American Diabetes Association GENNID (Genetics of NIDDM) Study Cohort. Diabetes 58: 268-274.

37. Min J, Kyung KY, Cipriani PG, Kang M, Khersonsky SM, et al. (2007) Forward chemical genetic approach identifies new role for GAPDH in insulin signaling. Nat Chem Biol 3: 55-59.

38. Cheng CY, Kao WH, Patterson N, Tandon A, Haiman CA, et al. (2009) Admixture mapping of 15,280 African Americans identifies obesity susceptibility loci on chromosomes 5 and X. PLoS Genet 5: e1000490.

39. Genovese G, Friedman DJ, Ross MD, Lecordier L, Uzureau P, et al. (2010) Association of trypanolytic ApoL1 variants with kidney disease in African Americans. Science 329: 841-845.

40. Patterson N, Hattangadi N, Lane B, Lohmueller KE, Hafler DA, et al. (2004) Methods for high-density admixture mapping of disease genes. Am J Hum Genet 74: 979-1000.

41. Cheng CY, Reich D, Coresh J, Boerwinkle E, Patterson N, et al. (2010) Admixture mapping of obesity-related traits in African Americans: the Atherosclerosis Risk in Communities (ARIC) Study. Obesity (Silver Spring) 18: 563-572.

42. Braveman PA, Cubbin C, Egerter S, Chideya S, Marchi KS, et al. (2005) Socioeconomic status in health research: one size does not fit all. JAMA 294: 2879-2888.

43. The ARIC Investigators (1989) The Atherosclerosis Risk in Communities (ARIC) Study: design and objectives. The ARIC investigators. Am J Epidemiol 129: $687-702$. 
44. Taylor HA, Jr. (2003) Establishing a foundation for cardiovascular disease research in an African-American community-the Jackson Heart Study. Ethn Dis 13: 411-413.

45. Taylor HA, Jr., Wilson JG, Jones DW, Sarpong DF, Srinivasan A, et al. (2005) Toward resolution of cardiovascular health disparities in African Americans: design and methods of the Jackson Heart Study. Ethn Dis 15: S6-17.

46. Wilson JG, Rotimi CN, Ekunwe L, Royal CD, Crump ME, et al. (2005) Study design for genetic analysis in the Jackson Heart Study. Ethn Dis 15: S6-37.

47. Kolonel LN, Henderson BE, Hankin JH, Nomura AM, Wilkens LR, et al. (2000) A multiethnic cohort in Hawaii and Los Angeles: baseline characteristics. Am J Epidemiol 151: 346-357.

48. Selvin E, Coresh J, Zhu H, Folsom A, Steffes MW (2010) Measurement of HbAlc from stored whole blood samples in the Atherosclerosis Risk in Communities study. J Diabetes 2: 118-124.

49. Carpenter MA, Crow R, Steffes M, Rock W, Heilbraun J, et al. (2004) Laboratory, reading center, and coordinating center data management methods in the Jackson Heart Study. Am J Med Sci 328: 131-144.

50. U.S. Department of Health, Education, and Welfare (1976) Publication No. (CDC) 77-8330. National Glucose Reference Method.

51. American Diabetes Association (2009) Diagnosis and classification of diabetes mellitus. Diabetes Care 32 Suppl 1: S62-S67.
52. Waters KM, Henderson BE, Stram DO, Wan P, Kolonel LN, et al. (2009) Association of diabetes with prostate cancer risk in the multiethnic cohort. Am J Epidemiol 169: 937-945.

53. Deo RC, Patterson N, Tandon A, McDonald GJ, Haiman CA, et al. (2007) A High-Density Admixture Scan in 1,670 African Americans with Hypertension. PLoS Genet 3: e196.

54. Bureau of the Census (1982) 1980 Census of population: classified index of industries and occupations.

55. Smith MW, Patterson N, Lautenberger JA, Truelove AL, McDonald GJ, et al. (2004) A high-density admixture map for disease gene discovery in african americans. Am J Hum Genet 74: 1001-1013.

56. Reich D, Patterson N, De Jager PL, McDonald GJ, Waliszewska A, et al. (2005) A whole-genome admixture scan finds a candidate locus for multiple sclerosis susceptibility. Nat Genet 37: 1113-1118.

57. Cheng CY, Reich D, Wong TY, Klein R, Klein BE, et al. (2010) Admixture mapping scans identify a locus affecting retinal vascular caliber in hypertensive African Americans: the Atherosclerosis Risk in Communities (ARIC) study. PLoS Genet 6: e1000908.

58. Reich D, Patterson N (2005) Will admixture mapping work to find disease genes? Philos Trans R Soc Lond B Biol Sci 360: 1605-1607. 